The Astrophysical Journal, 656:408-413, 2007 February 10

(C) 2007. The American Astronomical Society. All rights reserved. Printed in U.S.A.

\title{
DISCOVERY OF FIVE RECYCLED PULSARS IN A HIGH GALACTIC LATITUDE SURVEY
}

\author{
B. A. Jacoby, ${ }^{1,2}$ M. Bailes, ${ }^{3}$ S. M. Ord, ${ }^{3,4}$ H. S. Knight, ${ }^{3,5}$ and A. W. Hotan ${ }^{3,5}$ \\ Received 2005 August 26; accepted 2006 September 14
}

\begin{abstract}
We present five recycled pulsars discovered during a $21 \mathrm{~cm}$ survey of approximately $4150 \mathrm{deg}^{2}$ between $15^{\circ}$ and $30^{\circ}$ from the Galactic plane using the Parkes radio telescope. One new pulsar, PSR J1528-3146, has a $61 \mathrm{~ms}$ spin period and a massive white dwarf companion. Like many recycled pulsars with heavy companions, the orbital eccentricity is relatively high $(\sim 0.0002)$, consistent with evolutionary models that predict less time for circularization. The four remaining pulsars have short spin periods ( $3 \mathrm{~ms}<P<6 \mathrm{~ms}$ ); three of these have probable white dwarf binary companions and one (PSR J2010-1323) is isolated. PSR J1600-3053 is relatively bright for its dispersion measure of $52.3 \mathrm{pc} \mathrm{cm}^{-3}$ and promises good timing precision thanks to an intrinsically narrow feature in its pulse profile, resolvable through coherent dedispersion. In this survey, the recycled pulsar discovery rate was 1 per 4 days of telescope time or 1 per $600 \mathrm{deg}^{2}$ of sky. The variability of these sources implies that there are more millisecond pulsars that might be found by repeating this survey.
\end{abstract}

Subject headings: binaries: close — pulsars: general — stars: neutron — surveys

\section{INTRODUCTION}

Most pulsars are thought to descend from massive stars in the disk of the Galaxy, and as a result, the bulk of pulsar search efforts have historically been concentrated near the Galactic plane where the majority of pulsars, with their fairly short observable lifetimes, reside (e.g., Stokes et al. 1986; Clifton et al. 1992; Johnston et al. 1992; Manchester et al. 2001). Early surveys along the plane met with limited success when searching for recycled pulsars. The value of finding new recycled pulsars lies in their contribution to our understanding of the binary evolution processes (Bhattacharya \& van den Heuvel 1991), tests of general relativity (van Straten et al. 2001), and their use in a millisecond pulsar (MSP) timing array for the detection of low-frequency gravitational waves (Foster \& Backer 1990).

The pioneering survey of Wolszczan (1991) led to the discovery of two fascinating systems in a small area at high Galactic latitude. This success spawned several surveys at high Galactic latitudes, which yielded a large number of recycled pulsars (Bailes et al. 1994; Foster et al. 1995; Lorimer et al. 1995; Nicastro et al. 1995; Camilo et al. 1996). Simulations of the low-luminosity Galactic recycled pulsar population point to a more isotropic distribution than is expected for the more luminous long-period pulsars that are less subject to the deleterious effects of the interstellar medium (Johnston \& Bailes 1991). This difference occurs because recycled pulsars have had a longer time to migrate away from their birthplace in the Galactic disk, and their shorter periods strongly limit the distance to which they can be detected in the electron-rich Galactic plane. These facts, combined with the relative insensitivity to dispersion and lower sky temperatures afforded by highfrequency observations, suggested that a $21 \mathrm{~cm}$ survey for pulsars

\footnotetext{
1 Department of Astronomy, California Institute of Technology, Pasadena, CA.

Current address: Naval Research Laboratory, Washington, DC; bryan.jacoby@ nrl.navy.mil.

3 Centre for Astrophysics and Supercomputing, Swinburne University of Technology, Hawthorn, Australia; mbailes@astro.swin.edu.au, hknight@astro .swin.edu.au, ahotan@astro.swin.edu.au.

${ }^{4}$ Current address: School of Physics, University of Sydney, Sydney, NSW, Australia; ord@physics.usyd.edu.au.

5 Australia Telescope National Facility, Epping, NSW, Australia.
}

away from the Galactic plane would be extremely productive (Toscano et al. 1998). The success of the Swinburne Intermediate Latitude Pulsar Survey demonstrated the validity of this approach, discovering eight recycled pulsars in $2950 \mathrm{deg}^{2}$ at Galactic latitudes between $5^{\circ}$ and $15^{\circ}$ (Edwards \& Bailes 2001a, 2001b; Edwards et al. 2001). Recent reprocessing of the Parkes multibeam survey, which concentrated on the Galactic plane $\left(|b|<5^{\circ}\right)$ has also yielded millisecond and recycled pulsars in large numbers (Faulkner et al. 2004).

In this paper we describe five recycled pulsars discovered in this extension of the Swinburne Intermediate Latitude survey. In $\S 2$ we describe the survey parameters before discussing the five pulsars individually in $\S 3$, giving their timing solutions (where possible), pulse profiles, and derived parameters. In $\S 4$ we discuss our results.

\section{A HIGH-LATITUDE PULSAR SURVEY}

This survey was carried out using the 13 beam multibeam receiver on the Parkes $64 \mathrm{~m}$ radio telescope from 2001 January to 2002 December. This survey covered $\sim 4150 \mathrm{deg}^{2}$ in the region $-100^{\circ}<l<50^{\circ}, 15^{\circ}<|b|<30^{\circ}$. Relatively short 265 s integrations gave a sensitivity that is well matched to the expected scale height and luminosity distribution of the pulsar population (Cordes \& Chernoff 1997), and allowed us to complete the 7232 survey pointings in about 4 weeks of observing. The signals from each beam were processed and digitized by a $2 \times 96 \times 3 \mathrm{MHz}$ filter bank operating at a center sky frequency of $1374 \mathrm{MHz}$ and 1 bit sampled every $125 \mu \mathrm{s}$, providing good sensitivity to fast pulsars with low to moderate dispersion measures. This observing methodology is identical to that employed for the Swinburne Intermediate Latitude Pulsar Survey and differs from that of the Parkes Multibeam Pulsar Survey (Manchester et al. 2001) only in sampling period (125 vs. $250 \mu \mathrm{s}$ ) and integration time (265 vs. $2100 \mathrm{~s})$.

The resulting 2.4 TB of data were searched for pulsar-like signals using standard techniques with the 64 Compaq Alpha workstations at the Swinburne Centre for Astrophysics and Supercomputing, resulting in the discovery of 26 new pulsars. Full details of this survey will be described in a future paper (B. A. Jacoby et al., in preparation). Of these 26 new pulsars, 

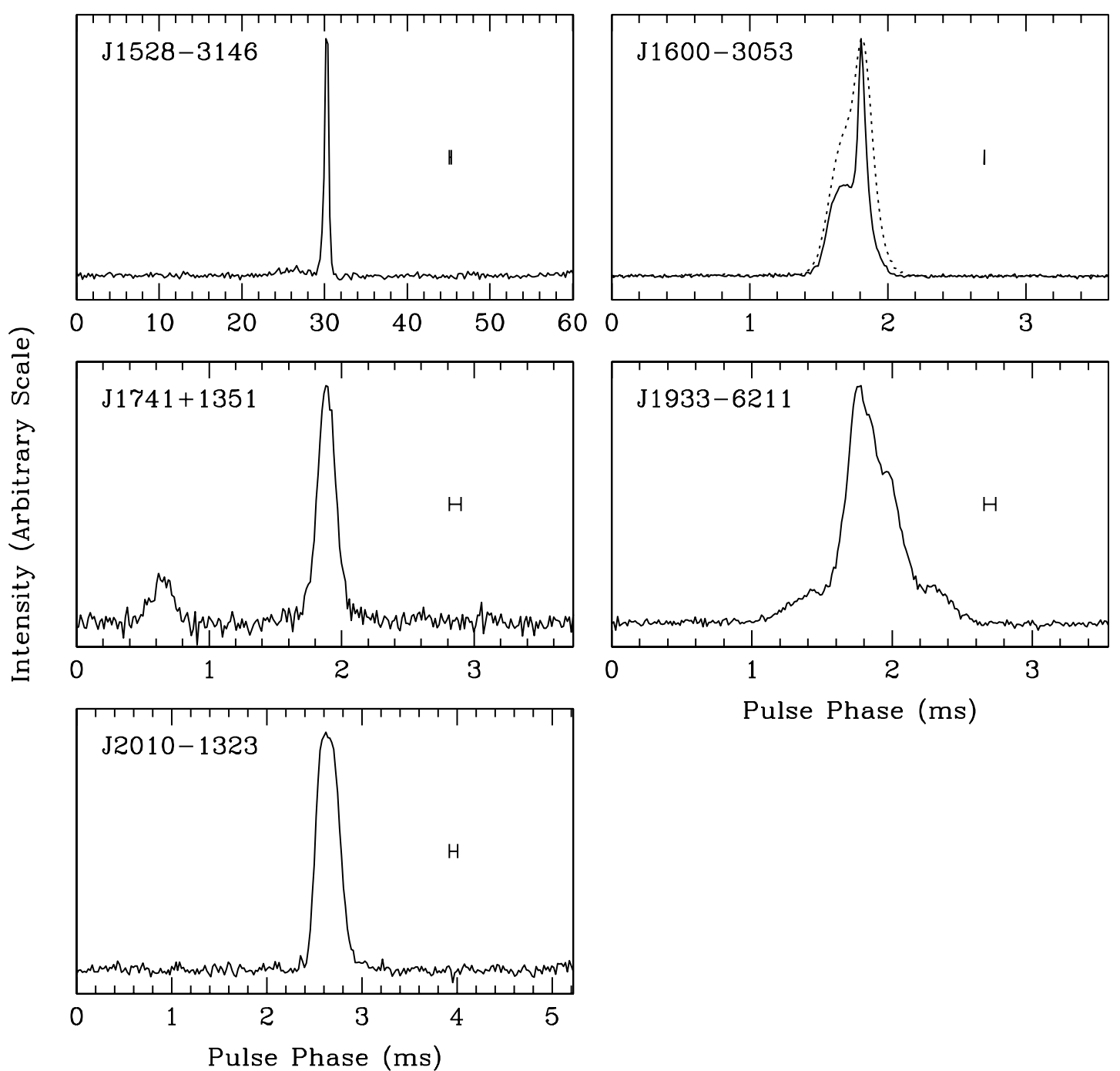

FIG. 1. - Average pulse profiles at $1.4 \mathrm{GHz}$. PSR J1600-3053 profile measured with CPSR2 (solid) and the $512 \times 0.5 \mathrm{MHz}$ filter bank (dotted), and J1741+1351 profile measured by the $96 \times 3 \mathrm{MHz}$ filter bank. The $512 \times 0.5 \mathrm{MHz}$ filter bank was used for all others. Horizontal bars represent the time resolution of the observing system arising from the differential dispersion within a filter-bank channel and the sampling interval, except for J1600-3053 where horizontal bar indicates $2 \mu$ s time resolution of the coherently dedispersed pulse profile.

seven are recycled. One of them, PSR J1909-3744, is an exceptionally interesting millisecond pulsar and has been reported elsewhere (Jacoby et al. 2003, 2005), and another, PSR J1738+ 0333, will be described in a latter paper (B. A. Jacoby et al., in preparation). The other five are described here.

\section{DISCOVERY AND TIMING OF FIVE RECYCLED PULSARS}

These five objects belong to the class of recycled pulsars with relatively weak magnetic fields and small spin-down rates. Four are MSPs with spin periods well under $10 \mathrm{~ms}$; the fifth has a longer spin period, but is still clearly recycled due to its large characteristic age $\left(\tau_{c}\right)$. One of the MSPs is isolated; the other pulsars are in binary systems with probable white dwarf companions. Average pulse profiles of the five pulsars are shown in Figure 1.

We have begun a systematic timing program at Parkes for these and other pulsars discovered in this survey, primarily using the $2 \times 512 \times 0.5 \mathrm{MHz}$ filter bank at $1390 \mathrm{MHz}$, with occasional observations using the $2 \times 256 \times 0.125 \mathrm{MHz}$ filter bank at $660 \mathrm{MHz}$ to determine the dispersion measure (DM).

We followed standard pulsar timing procedures: folded pulse profiles from individual observations were cross-correlated with a high signal-to-noise ratio $(\mathrm{S} / \mathrm{N})$ template profile to determine an average pulse time of arrival (TOA) corrected to UTC (NIST). The standard pulsar timing package TEMPO $^{6}$, along with the Jet Propulsion Laboratory's DE405 ephemeris, was used for all timing analysis. TOA uncertainties for each pulsar were multiplied by a factor between 1.12 and 1.65 to achieve reduced $\chi^{2} \simeq 1$. One of the new pulsars, PSR J1933-6211, has a very small orbital eccentricity $(e)$, giving rise to a strong covariance between the time of periastron $\left(T_{0}\right)$ and longitude of periastron $(\omega)$ in this system. For this pulsar, we have used the ELL1 binary model, which replaces $\omega, T_{0}$, and $e$ with the time of ascending node $\left(T_{\text {asc }}\right)$ and the Laplace-Lagrange parameters $e \sin \omega$ and $e \cos \omega$ (Lange et al. 2001). We have used the DD model (Damour \& Deruelle $1985,1986)$ for PSR J1528-3146 and PSR J1600-3053.

For PSR J1741+1351, orbital parameters were obtained by fitting a model to observed spin periods obtained from multiple observations with the 96 channel survey filter bank. The position reported here is the center of the survey beam in which the pulsar was discovered; timing analysis of this pulsar is ongoing and will be reported in a future paper (P. C. Freire et al., in preparation).

\footnotetext{
${ }^{6}$ See http://pulsar.princeton.edu/tempo.
} 
TABLE 1

Pulsar Parameters for J1528-3146 and J1600-3053

\begin{tabular}{|c|c|c|}
\hline Parameter $^{\mathrm{a}}$ & J1528-3146 & $\mathrm{J} 1600-3053$ \\
\hline R. A., $\alpha_{\mathrm{J} 2000.0}$ & $152834.9542(2)$ & $160051.90392(2)$ \\
\hline 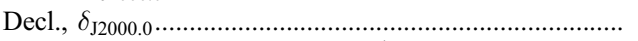 & $-314606.836(8)$ & $-305349.325(2)$ \\
\hline 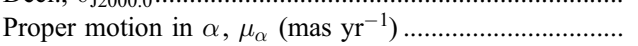 & $\ldots$ & $-0.91(51)$ \\
\hline 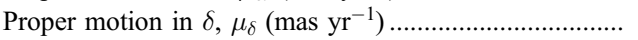 & & $-4.0(15)$ \\
\hline Pulse period, $P(\mathrm{~ms})$ & $60.82223035146(1)$ & $3.59792845222642(6)$ \\
\hline Reference epoch (MJD) & 52500.0 & 52500.0 \\
\hline Period derivative, $\dot{P}\left(10^{-20}\right) \ldots \ldots \ldots \ldots . . . .$. & 24.9(1) & $0.9479(4)$ \\
\hline Dispersion measure, $\mathrm{DM}\left(\mathrm{pc} \mathrm{cm}^{-3}\right) \ldots$ & $18.163(6)$ & $52.333(1)$ \\
\hline 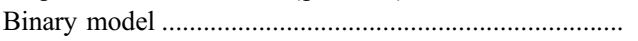 & DD & DD \\
\hline Binary period, $P_{b}$ (days) & $3.180345754(3)$ & $14.348457554(4)$ \\
\hline 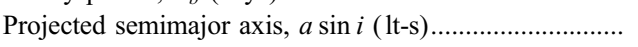 & $11.452324(5)$ & $8.8016571(4)$ \\
\hline Orbital eccentricity, $e$ & $0.000213(1)$ & $0.00017371(8)$ \\
\hline Longitude of periastron, $\omega(\mathrm{deg})$ & $296.83635 \pm 0.2$ & $181.768043 \pm 0.03$ \\
\hline Time of periastron, $T_{0}$ & $52502.4013744 \pm 0.002$ & $52506.3711244 \pm 0.001$ \\
\hline Weighted rms timing residual $(\mu \mathrm{s}) \ldots \ldots \ldots \ldots \ldots \ldots \ldots \ldots \ldots \ldots \ldots \ldots \ldots$ & 11.1 & 1.55 \\
\hline
\end{tabular}

\begin{tabular}{|c|c|c|}
\hline \multicolumn{3}{|c|}{ Derived Parameters } \\
\hline Minimum companion mass $m_{c} \min \left(M_{\odot}\right) \ldots \ldots \ldots \ldots \ldots \ldots \ldots \ldots$ & 0.94 & 0.20 \\
\hline Galactic longitude, $l(\mathrm{deg})$ & 337.94 & 344.09 \\
\hline Galactic latitude, $b$ (deg). & 20.22 & 16.45 \\
\hline DM-derived distance, $d(\mathrm{kpc})^{\mathrm{b}} .$. & 0.80 & 1.53 \\
\hline 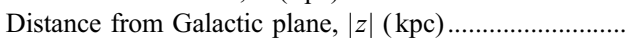 & 0.28 & 0.43 \\
\hline 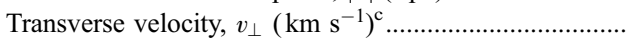 & $\ldots$ & $30_{-15}^{+16}$ \\
\hline 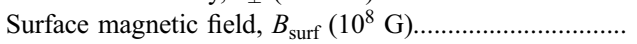 & 39.3 & $1.8^{\mathrm{d}}$ \\
\hline 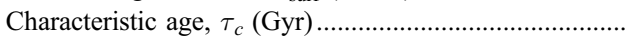 & 3.9 & $6.2^{\mathrm{d}}$ \\
\hline Pulse FWHM, $w_{50}(\mathrm{~ms})$ & 0.59 & 0.079 \\
\hline Pulse width at $10 \%$ peak, $w_{10}(\mathrm{~ms})$ & 1.29 & 0.41 \\
\hline Discovery $\mathrm{S} / \mathrm{N}$ & 28.0 & 16.7 \\
\hline 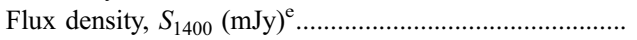 & 1.1 & 3.2 \\
\hline
\end{tabular}

Nоте.-Units of right ascension are hours, minutes, and seconds, and units of declination are degrees, arcminutes, and arcseconds.

${ }^{a}$ Figures in parenthesis are uncertainties in the last digit quoted. Uncertainties are calculated from twice the formal error produced by TEMPO.

${ }^{\mathrm{b}}$ From the model of Cordes \& Lazio (2002).

${ }^{\mathrm{c}}$ Stated uncertainty in transverse velocity is based only on uncertainty in proper motion; the distance is taken as exact.

${ }^{\mathrm{d}}$ Corrected for secular acceleration based on measured proper motion and estimated distance (Shklovskii 1970).

${ }^{\text {e }}$ Flux density estimated from observed S/N and nominal system parameters, except for PSR J1600-3053 from Ord et al. (2004).

Astrometric, spin, binary, and derived parameters for all pulsars are given in Tables 1 and 2 . Timing residuals for the four pulsars with timing solutions are shown in Figure 2.

\subsection{PSR J1528-3146: A Recycled Pulsar with a Massive Companion}

PSR J1528-3146 has the longest spin period $(P=61 \mathrm{~ms})$ of the five new pulsars reported here. The minimum companion mass (obtained from the mass function by assuming an edge-on orbit and a pulsar mass of $1.35 M_{\odot}$ ) is $0.94 M_{\odot}$; the system's circular orbit suggests that the companion must be a $\mathrm{CO}$ or $\mathrm{ONeMg}$ white dwarf. Only a handful of such intermediate-mass binary pulsar (IMBP) systems are known (Edwards \& Bailes 2001b; Camilo et al. 2001). Of all low-eccentricity binary pulsars, this object has the second highest minimum companion mass and fifth highest projected orbital velocity. The orbital parameters of PSR J15283146 are broadly similar to those of PSR J1157-5112, the loweccentricity binary pulsar with the most massive white dwarf companion (Edwards \& Bailes 2001a). The orbital eccentricity $(\sim 2 \times$ $10^{-4}$ ) is high compared to recycled pulsars with similar orbital periods. The large mass of the companion suggests a shorter duration spin-up phase, leaving less time to circularize the post- supernova orbit and transfer mass to the pulsar. At the dispersion measure of $18 \mathrm{pc} \mathrm{cm}^{-3}$, the implied distance is only about $1 \mathrm{kpc}$. We have detected a potential optical counterpart at the pulsar timing position with $R \sim 24.2$. As with other optically detected IMBP companions, this is brighter than expected given the characteristic age of the pulsar, suggesting that the spin-down age overestimates the cooling age of the white dwarf and hence the time since the end of mass transfer in the system (Jacoby et al. 2006).

\subsection{PSR J1600-3053: A High Precision Timing LMBP}

Our timing results obtained with the Parkes filter bank for this $3.6 \mathrm{~ms}$ pulsar have a weighted rms residual of $1.55 \mu$ s over $3 \mathrm{yr}$. Using the Caltech-Parkes-Swinburne Recorder II (CPSR2; see Jacoby 2005), a wide-bandwidth coherent dedispersion back end at Parkes, Ord et al. (2006) have achieved an rms residual of only $650 \mathrm{~ns}$ for this pulsar; measurement of the Shapiro delay constrains the orbital inclination to be between $59^{\circ}$ and $70^{\circ}$ (95\% confidence). This pulsar will be an important part of pulsar timing array experiments aimed at detecting low-frequency gravitational waves emitted by coalescing supermassive black hole binaries (Jaffe \& Backer 2003). 
TABLE 2

Pulsar Parameters for J1741+1351, J1933-6211, and J2010-1323

\begin{tabular}{|c|c|c|c|}
\hline Parameter $^{\mathrm{a}}$ & $\mathrm{J} 1741+1351$ & J1933-6211 & $\mathrm{J} 2010-1323$ \\
\hline R. A., $\alpha_{\mathrm{J} 2000.0} \cdots .$. & $174137 \pm 1^{\prime}$ & $193332.4272(3)$ & $201045.9196(2)$ \\
\hline Decl., $\delta_{\mathrm{J} 2000.0} \ldots \ldots \ldots . .$. & $+135441 \pm 14^{\prime}$ & $-621146.881(4)$ & $-132356.027(6)$ \\
\hline Pulse period, $P(\mathrm{~ms})$. & $3.7471544(6)$ & $3.543431438847(1)$ & $5.223271015190(1)$ \\
\hline Reference epoch (MJD).. & $\ldots$ & 53000.0 & 52500.0 \\
\hline Period derivative, $\dot{P}\left(10^{-20}\right) \ldots \ldots \ldots$ & & $0.37(1)$ & $0.482(7)$ \\
\hline 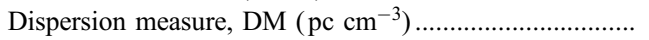 & $24.0(3)$ & $11.499(7)$ & $22.160(2)$ \\
\hline 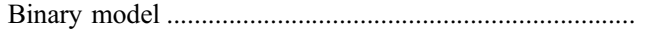 & $\ldots$ & ELL1 & $\ldots$ \\
\hline Binary period, $P_{b}$ (days) & $16.335(2)$ & $12.81940650(4)$ & $\ldots$ \\
\hline 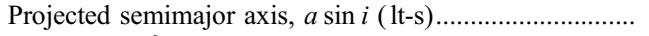 & $11.03(6)$ & $12.281575(3)$ & $\ldots$ \\
\hline$e \sin \omega\left(\times 10^{-6}\right)$ & $\ldots$ & $1.1(4)$ & $\ldots$ \\
\hline$e \cos \omega\left(\times 10^{-6}\right)$ & .. & $-0.55(50)$ & .. \\
\hline 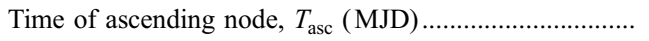 & $52846.22(1)$ & $53000.4951005(5)$ & $\ldots$ \\
\hline Weighted rms timing residual $(\mu \mathrm{s})$ & $\ldots$ & 6.06 & 4.25 \\
\hline
\end{tabular}

Derived Parameters

\begin{tabular}{|c|c|c|c|}
\hline 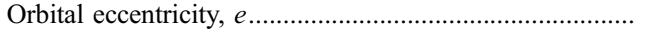 & ... & $0.0000013(4)$ & ... \\
\hline 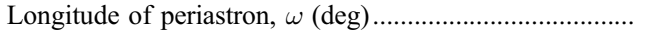 & $\ldots$ & $115.93036 \pm 22$ & .. \\
\hline Time of periastron, $T_{0}(\mathrm{MJD})$ & ... & $53004.6233183 \pm 0.8$ & .. \\
\hline Minimum companion mass $m_{c} \min \left(M_{\odot}\right) \ldots \ldots \ldots \ldots \ldots \ldots \ldots \ldots \ldots$ & 0.24 & 0.32 & .. \\
\hline 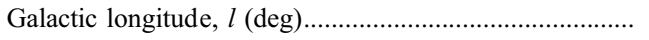 & 37.94 & 334.43 & 29.45 \\
\hline Galactic latitude, $b$ (deg) & 21.64 & -28.63 & -23.54 \\
\hline 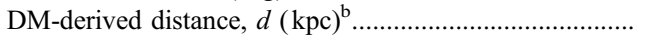 & 0.91 & 0.52 & 1.02 \\
\hline 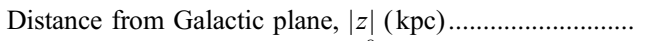 & 0.34 & 0.25 & 0.41 \\
\hline 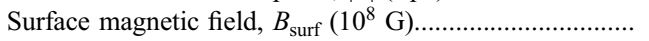 & $\ldots$ & 1.2 & 1.6 \\
\hline 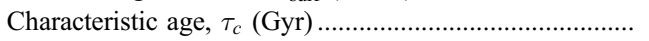 & $\ldots$ & 15 & 17 \\
\hline Pulse FWHM, $w_{50}(\mathrm{~ms})$ & 0.16 & 0.36 & 0.28 \\
\hline Pulse width at $10 \%$ peak, $w_{10}(\mathrm{~ms})$ & 0.32 & 1.03 & 0.44 \\
\hline Discovery $\mathrm{S} / \mathrm{N}$ & 10.7 & 22.9 & 12.4 \\
\hline 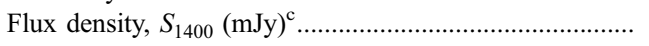 & 0.93 & 2.3 & 1.6 \\
\hline
\end{tabular}

\subsection{PSR J1741+1351: An LMBP with Strong Scintillation}

This $3.7 \mathrm{~ms}$ LMBP scintillates very strongly at $1.4 \mathrm{GHz}$, frequently making it undetectable in reasonable $(\sim 15$ minute $)$ integration times at Parkes. In fact, four attempts were required to confirm this pulsar candidate, highlighting why repeating this survey would no doubt turn up other pulsars that are, on average, below the nominal survey sensitivity limit. This strong scintillation partly explains why this pulsar was not discovered in previous Arecibo surveys. We have not yet obtained a phaseconnected timing solution for this pulsar. The minimum companion mass of $0.24 M_{\odot}, 16$ day orbital period, and inconsistent flux make it uninteresting for tests of general relativity with current instrumentation.

\subsection{PSR J1933-6211: An MSP with an Edge-on Orbit?}

This short period ( $3.4 \mathrm{~ms}$ ) pulsar's companion has a minimum mass of $0.32 M_{\odot}$, somewhat higher than the typical LMBP. The only other disk pulsars with companions of at least $0.3 M_{\odot}$ and spin periods shorter than $10 \mathrm{~ms}$ are PSR J2019+2425 (Nice et al. $1993 ; P=3.9 \mathrm{~ms})$ with a much longer orbital period $\left(P_{b}=76\right.$ vs. 13 days), and PSR J1757-5322 (Edwards \& Bailes 2001b; $P=8.9 \mathrm{~ms}$ ) and PSR J1435-6100 (Camilo et al. 2001; $P=$ $9.3 \mathrm{~ms})$ with substantially more compact orbits $\left(P_{b}=1.4\right.$ and 0.45 days, respectively). This may mean that this pulsar has a nearly edge-on orbit or represents a limit to accretion spin-up given the mass of the companion.

\subsection{PSR J2010-1323: Isolated MSP}

This $5.2 \mathrm{~ms}$ pulsar is the only isolated MSP found in this survey. Approximately one fourth of recycled pulsars not associated with globular clusters are isolated. The rms timing residual is $4 \mu \mathrm{s}$ with a $256 \mathrm{MHz}$ filter bank system, and there is no evidence of any planetary system like that surrounding the planet pulsar PSR B1257+12. With hour-long integrations and coherent dedispersion back end, we anticipate that a weighted rms residual of approximately $1 \mu \mathrm{s}$ will be possible for this pulsar with the Parkes telescope and perhaps half of that with the $100 \mathrm{~m}$ Green Bank Telescope.

\section{DISCUSSION}

This survey has once again demonstrated the efficacy of the Parkes $64 \mathrm{~m}$ telescope's multibeam receiver for discovering recycled pulsars. The recycled pulsar discovery rate of 1 per $600 \mathrm{deg}^{2}$ is comparable to that of Edwards et al. (2001). However, only one new recycled pulsar was discovered more than $25^{\circ}$ and less than $30^{\circ}$ from the plane, compared to the five between $5^{\circ}$ and $10^{\circ}$ from the plane, which may indicate that the density of MSPs detectable with this back end and telescope combination drops off considerably when more than $25^{\circ}$ from the Galactic plane.

The ratio of the number of recycled pulsars still in binaries to those that are now single puts a limit on the fraction of systems where recycling goes out of control, resulting in a solitary pulsar either because the pulsar gets too close to the companion and 


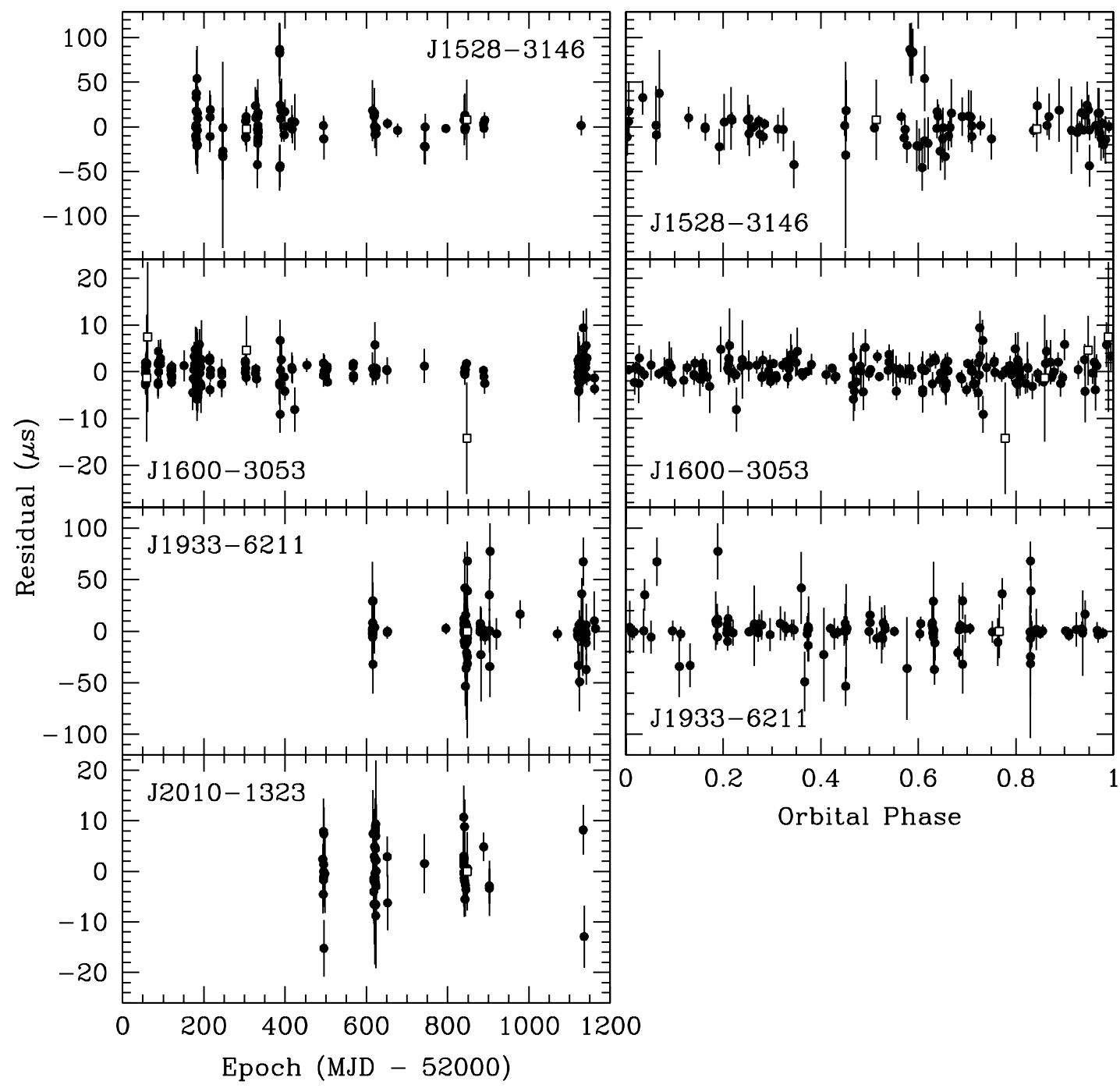

FIG. 2.-Timing residuals plotted vs. observation epoch (left column) and orbital phase (right column) for pulsars with phase-connected timing solutions. Filled circles represent observations at $1390 \mathrm{MHz}$, open squares represent $600 \mathrm{MHz}$ observations.

ablates it with its strong wind, or because it tidally disrupts the companion completely. In this survey six of the seven recycled pulsars are in binary systems, which in itself suggests that in the majority of cases recycling is not fatal to the donor star. Thanks to the Parkes multibeam surveys, the disk population of millisecond pulsars is now becoming large enough for us to search for trends that might explain why some millisecond pulsars are single and others are not, and which parameters are correlated. The recycled pulsars found in this survey have reinforced the previously observed trend of pulsars with high-mass companions having longer spin periods and higher eccentricities than those with low-mass companions.

Large-scale uniform surveys allow us to model the underlying populations' properties, including the $z$-scale height of MSPs. There are 11 field MSPs (defined for our purposes as having $P<20 \mathrm{~ms}$ ) with $|z|>0.5 \mathrm{kpc}$ in the ATNF Pulsar Catalogue ${ }^{7}$ (Manchester et al. 2005), with DM-based distances provided by the NE2001 model (Cordes \& Lazio 2002) for pulsars without direct distance estimates. Of these pulsars, four were discovered by the Parkes $70 \mathrm{~cm}$ survey (Manchester et al. 1996; Lyne et al. 1998), and none by the Swinburne surveys (although we would have expected these surveys to have similar sensitivities to high-

\footnotetext{
7 See http://www.atnf.csiro.au/research/pulsar/psrcat/.
}

$|z|$ pulsars). The Arecibo surveys that are responsible for most of the rest are difficult to model. To investigate the implications of the large-area Parkes surveys we simulated a population of 100,000 MSPs with a wide range in luminosity and a scale height of $500 \mathrm{pc}$. Our simulation predicted that half of all discoveries should have $|z|>0.5 \mathrm{kpc}$ for the Parkes $70 \mathrm{~cm}$ and the Swinburne surveys. In reality, only about $13 \%$ of the MSPs detected have $|z|>0.5 \mathrm{kpc}$, which suggests that either $0.5 \mathrm{kpc}$ is an overestimate of their scale height, or our modeling of their luminosities is incorrect. We note that somewhat different results are obtained using the older Taylor \& Cordes (1993) Galactic electron model to estimate distances. Based on this model, there are 17 MSPs with $|z|>0.5 \mathrm{kpc}$, five of which were discovered in the Parkes $70 \mathrm{~cm}$ survey and five of which were discovered by the Swinburne surveys. This suggests that values of the $z$ scale height from previous population studies based on the older distance model, such as the $0.65_{-0.12}^{+0.16} \mathrm{kpc}$ Gaussian scale height of Cordes \& Chernoff (1997) will be revised downward by the use of the new Galactic electron model.

Again restricting ourselves to disk pulsars with $P<20 \mathrm{~ms}$, we find 15 isolated MSPs and 44 in binary systems. The distribution of height above the Galactic plane is shown for these two populations in Figure 3. The rms deviation from $z=0$ is $240 \pm 40 \mathrm{pc}$ for isolated MSPs and $450 \pm 50 \mathrm{pc}$ for binary MSPs (if we consider 


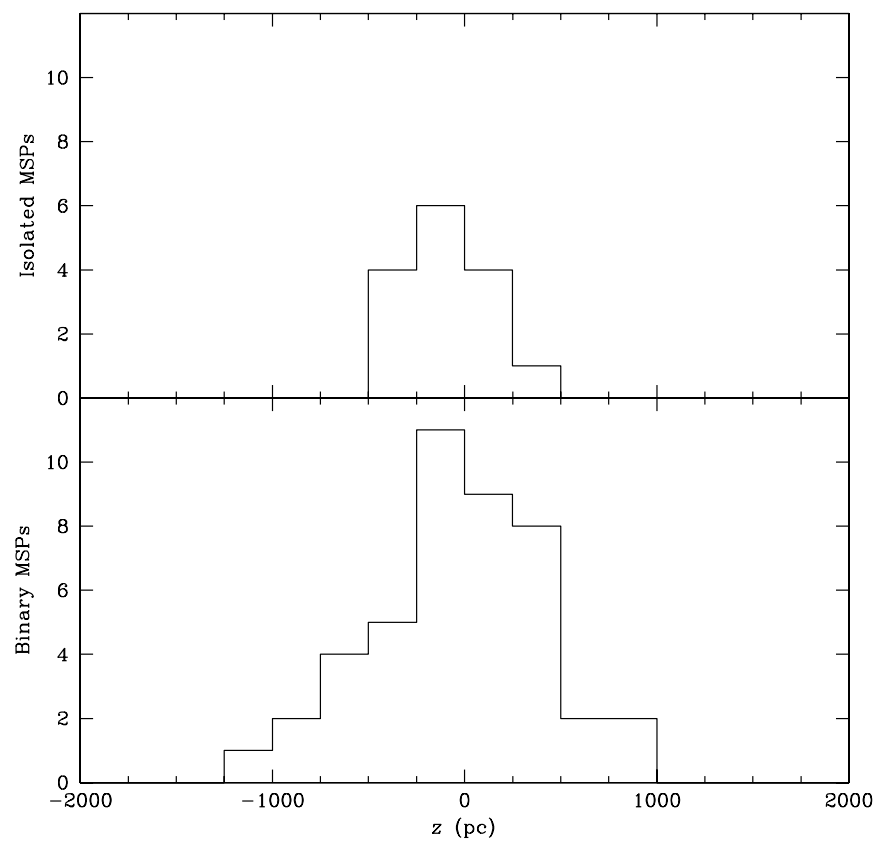

FIG. 3.- Histograms of height above the Galactic plane for isolated (top) and binary (bottom) field MSPs.

only pulsars with $P<10 \mathrm{~ms}$ the isolated sample is unchanged, while the binary sample drops to 37 objects with rms in $z$ of $470 \pm 60 \mathrm{pc}$ ). These results are similar to those obtained by Lommen et al. (2006) using a sample of 9 isolated and 20 binary pulsars with $P<10 \mathrm{~ms}$.
The median distance to the isolated and binary MSPs in our sample is $960 \mathrm{pc}$ and $1240 \mathrm{pc}$, respectively, regardless of the period cutoff used. The difference between these two populations is less than that noted by Lommen et al. (2006), who found more than a factor of 2 difference in their sample. This result may suggest that the luminosity distributions of isolated and binary MSPs are not as different as previously thought, with implications for the birth velocity distributions required by the different observed scale heights. A flux-monitoring campaign on a large population of MSPs, including those discovered here, should enable direct investigation of the tantalizing scale height-luminosity question for isolated and binary MSPs. If the isolated MSPs really do have lower luminosities we would expect them to have had a different accretion history from those still in binary systems. If it is the tight binary MSPs that evolve into isolated MSPs through either ablation or tidal destruction of their companions, then we might expect different underlying velocity distributions and therefore different scale heights for the isolated and binary populations.

We thank R. Edwards for invaluable help with pulsar search software. The Parkes telescope is part of the Australia Telescope, which is funded by the Commonwealth of Australia for operation as a National Facility managed by CSIRO. B. A. J. thanks NSF and NASA for supporting this research. B. A. J. holds a National Research Council Research Associateship Award at the Naval Research Laboratory (NRL). Basic research in radio astronomy at NRL is supported by the Office of Naval Research.
Bailes, M., et al. 1994, ApJ, 425, L41

Bhattacharya, D., \& van den Heuvel, E. P. J. 1991, Phys. Rep., 203, 1

Camilo, F., Nice, D. J., \& Taylor, J. H. 1996, ApJ, 461, 812

Camilo, F., et al. 2001, ApJ, 548, L187

Clifton, T. R., Lyne, A. G., Jones, A. W., McKenna, J., \& Ashworth, M. 1992, MNRAS, 254, 177

Cordes, J. M., \& Chernoff, D. F. 1997, ApJ, 482, 971

Cordes, J. M., \& Lazio, T. J. W. 2002, preprint (astro-ph/0207156)

Damour, T., \& Deruelle, N. 1985, Ann. Inst. H. Poincaré (Phys. Théor.), 43, 107 1986, Ann. Inst. H. Poincaré (Phys. Théor.), 44, 263

Edwards, R. T., \& Bailes, M. 2001a, ApJ, 547, L37 2001b, ApJ, 553, 801

Edwards, R. T., Bailes, M., van Straten, W., \& Britton, M. C. 2001, MNRAS, 326,358

Faulkner, A. J., et al. 2004, MNRAS, 355, 147

Foster, R. S., \& Backer, D. C. 1990, ApJ, 361, 300

Foster, R. S., Cadwell, B. J., Wolszczan, A., \& Anderson, S. B. 1995, ApJ, 454, 826

Jacoby, B. A. 2005, Ph.D. thesis, California Institute of Technology

Jacoby, B. A., Bailes, M., van Kerkwijk, M. H., Ord, S., Hotan, A., Kulkarni, S. R., \& Anderson, S. B. 2003, ApJ, 599, L99

Jacoby, B. A., Chakrabarty, D., van Kerkwijk, M. H., Kulkarni, S. R., \& Kaplan, D. L. 2006, ApJ, 640, L183

Jacoby, B. A., Hotan, A., Bailes, M., Ord, S., \& Kulkarni, S. R. 2005, ApJ, 629, L113

Jaffe, A. H., \& Backer, D. C. 2003, ApJ, 583, 616

\section{REFERENCES}

Johnston, S., \& Bailes, M. 1991, MNRAS, 252, 277

Johnston, S., Lyne, A. G., Manchester, R. N., Kniffen, D. A., D’Amico, N., Lim, J., \& Ashworth, M. 1992, MNRAS, 255, 401

Lange, C., Camilo, F., Wex, N., Kramer, M., Backer, D., Lyne, A., \& Doroshenko, O. 2001, MNRAS, 326, 274

Lommen, A. N., Kipphorn, R. A., Nice, D. J., Splaver, E. M., Stairs, I. H., \& Backer, D. C. 2006, ApJ, 642, 1012

Lorimer, D. R., et al. 1995, ApJ, 439, 933

Lyne, A. G., et al. 1998, MNRAS, 295, 743

Manchester, R. N., Hobbs, G. B., Teoh, A., \& Hobbs, M. 2005, AJ, 129, 1993

Manchester, R. N., et al. 1996, MNRAS, 279, 1235

2001, MNRAS, 328, 17

Nicastro, L., Lyne, A. G., Lorimer, D. R., Harrison, P. A., Bailes, M., \& Skidmore, B. D. 1995, MNRAS, 273, L68

Nice, D. J., Taylor, J. H., \& Fruchter, A. S. 1993, ApJ, 402, L49

Ord, S. M., Jacoby, B. A., Hotan, A. W., \& Bailes, M. 2006, MNRAS, 371, 337

Ord, S. M., van Straten, W., Hotan, A. W., \& Bailes, M. 2004, MNRAS, 352, 804

Shklovskii, I. S. 1970, Soviet Astron., 13, 562

Stokes, G. H., Segelstein, D. J., Taylor, J. H., \& Dewey, R. J. 1986, ApJ, 311, 694

Taylor, J. H., \& Cordes, J. M. 1993, ApJ, 411, 674

Toscano, M., Bailes, M., Manchester, R., \& Sandhu, J. 1998, ApJ, 506, 863 van Straten, W., Bailes, M., Britton, M., Kulkarni, S. R., Anderson, S. B., Manchester, R. N., \& Sarkissian, J. 2001, Nature, 412, 158

Wolszczan, A. 1991, Nature, 350, 688 